\title{
The need for pharmaceutical care in an intensive care unit at a teaching hospital in South Africa
}

\author{
E Bronkhorst, ${ }^{1}$ BPharm, MSc (Med) Pharmacy; N Schellack, ${ }^{1}$ BPharm, PhD Pharmacy; A G S Gous, ${ }^{1}$ BPharm, Pharm D; \\ J P Pretorius, ${ }^{2}$ MB ChB, MMed (Surg), FCS (SA) \\ ${ }^{1}$ Department of Pharmacy, University of Limpopo, Medunsa Campus, Ga-rankuwa, South Africa \\ ${ }^{2}$ Department of Critical Care, Medical School, Faculty of Health Sciences, University of Pretoria; and Steve Biko Academic Hospital, Pretoria, \\ South Africa
}

Corresponding author: E Bronkhorst (elmien.bronkhorst@ul.ac.za)

Background. The role of the pharmacist has evolved over the last 2 decades beyond traditional functions such as stock control and dispensing.

Objectives. To describe the functions performed by a clinical pharmacist while based in a surgical and trauma intensive care unit of a teaching hospital.

Methods. An operational research study that included indications of programme success was conducted. Interventions to assess therapy and achieve definite outcomes to satisfy patients' medicine needs were documented for 51 patients over a study period of 8 weeks. Results. A total of 181 interventions were suggested by the pharmacist, with 127 (70\%) accepted and implemented by the medical and nursing staff of the unit. The most frequent interventions were related to: untreated medical conditions (15.5\%), appropriate therapy or course (13.8\%), investigations indicated or outstanding (12.2\%), and inappropriate doses and dosing frequency (11\%). Interventions were also made regularly to address system errors or non-compliance and factors hindering therapeutic effect. Of the $250 \mathrm{~h}$ the pharmacist spent in the ward, most time was used for pharmaceutical care (28\%) and ward rounds (21\%) with members of the multidisciplinary team. Conclusions. The study results demonstrated that a clinical pharmacist's contribution to patient care at ward level resulted in improved monitoring of pharmacotherapy. Medicine-related problems were identified and addressed.

S Afr J Crit Care 2014;30(2):41-44. DOI:10.7196/SAJCC.194

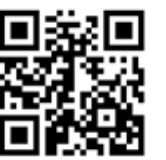

Pharmacy entered the 20th century in the social role of the apothecary, i.e. preparing and selling medicinal drugs. A pharmacist's main obligation was to ensure that the medicines he or she sold were pure, and to provide a good service to customers. ${ }^{[1]}$

Clinical pharmacy practice was born in the 1960 s and evolved to the responsible provision of medicine-related patient care in the $1980 \mathrm{~s}^{[2]}$ The act of performing clinical pharmaceutical interventions by the pharmacist as part of a healthcare team is called pharmaceutical care. ${ }^{[3]}$ Clinical pharmacy interventions and pharmaceutical care can be defined as the process of identifying a medicine-related problem and making a recommendation in an attempt to prevent or resolve it.

According to the Minnesota model, the first step for pharmaceutical care is to identify a patient's medicine-related needs. ${ }^{[4]}$ Categories of medicine-related needs or potential pharmaceutical interventions included are as follows: ${ }^{[4]}$

- New medicine therapy needed

- Unnecessary or wrong medicine therapy

- Dosage too low/high

- Adverse medicine reaction

- Compliance problem.

The effects or benefits of the provision of pharmaceutical care are measured to see whether a positive difference is made to the patient's clinical, human rights and economic outcomes. ${ }^{[5]}$ Benefits of pharmaceutical care can be derived by the patient, the healthcare team, the pharmacy profession and the health system. ${ }^{[5]}$
Currently in South Africa (SA), according to Schellack and Gous, ${ }^{[6]}$ for a pharmacist to be effective in a healthcare team, he or she must move into the wards where drugs are prescribed and administered. They also noted that doctors' ward rounds were a good training ground for ward pharmacists and an effective way of building relationships with prescribers. Through pharmaceutical care, the clinical pharmacist can play a role in concurrent review of prescribing patterns, providing feedback to the prescriber and educating providers and the public. ${ }^{[7]}$

Many pharmacy managers see ward rounds as time consuming and taking the pharmacist away from the dispensing practice. ${ }^{[6]}$ Combined with the low staff levels of pharmacists, suboptimal use of technical support staff and a lack of trained pharmacists, a low pharmacist presence in the wards has resulted. ${ }^{[6]}$

At the time of the current research, a clinical pharmacist was not part of routine care in medication monitoring to evaluate prescribing patterns or identify potential or actual medication errors in the surgical and trauma intensive care unit (ICU) at Steve Biko Academic Hospital. The study was initiated to address the need for pharmaceutical care and to evaluate the effect of the presence of a pharmacist in the ICU.

\section{Methods Research setting}

The study was conducted at Steve Biko Academic Hospital, an 832bed hospital with 53 ICU beds and 21 high-care beds, in Pretoria, Gauteng. The study site was the 12-bed surgical and trauma ICU. All the patients admitted to the unit during the 8-week study period, from Monday to Friday, were included in the study. 


\section{Study design}

This was a cross-sectional, operational research study. Indicators of programme success, such as improving the quality of service by monitoring medication errors and adverse effects, and adding new service components such as antimicrobial stewardship and drafting and implementation of antimicrobial guidelines, was an important part of the study design. The data were collected prospectively for 8 weeks. Quantitative aspects included patient demographics, clinical data, the number of different types of interventions as portrayed in the pharmaceutical care problem list, time spent on activities at ward level, the cost implications of pharmaceutical care interventions, and prescribing patterns of antimicrobials.

\section{Data collection instrument}

The data collection instrument comprised a validated pharmaceutical care form, which has been used in multiple previous clinical studies in SA. ${ }^{[8-10]}$ The form contained a patient database sheet to collect demographic data and a section to evaluate the patient's medication therapy, and to identify potential and actual drug problems.

\section{Data collection process}

Pharmaceutical care was rendered on a daily basis to all patients admitted to the ICU. Patient prescriptions and clinical data were evaluated by the pharmacist, whereafter necessary interventions were discussed with doctors during the daily ward rounds. The interventions and whether they were successful or not were recorded on the pharmaceutical care form. Interventions included discontinuation of antibiotics after completing a course, or reducing the antibiotic dose according to guidelines.

Medicine cost was calculated by determining the difference between the cost of medication used the day before and the day after an intervention. This amount was a direct calculation and only for the cost of one day after the intervention was performed. No calculations were made for indirect cost implications or length of ICU stay.

\section{Validity and reliability}

A pilot study was conducted prior to the main study to verify the appropriateness of the data collection instrument, in order to further enhance the reliability and validity of the data collection. Interventions towards patient therapy were based on the literature and discussed with medical practitioners to eliminate researcher bias.

\section{Ethical considerations}

Permission to conduct the study was obtained from the hospital management and institutional ethics review boards of the University of Limpopo (Medunsa campus) (MREC/H/15/2011) and the University of Pretoria (226/2010). Informed consent was obtained from patients, family members or legal guardians.

\section{Results}

A total of 51 patients was included in the study.

\section{Demographic information}

Of the 51 study patients, 35 were male and 16 female. The age of the patients ranged from 12 to 86 years, with a mean of 44.9 years. The average length of stay (LOS) in the ICU was 8.7 days (range 1 - 46). Patients older than 60 years had a longer LOS (10 - 12 days) in the ICU compared with patients younger than 30 years ( 1 - 3 days). Only $13(26 \%)$ of the 51 study patients' HIV status was known; 6 were HIVpositive, while 7 were HIV-negative. Of the 6 patients who were HIVpositive, 3 were previously receiving antiretroviral treatment, while 2 were prescribed antiretroviral treatment while admitted. One patient did not want her status disclosed and therefore could not be treated.

Table 1. Medication used according to ATC classification

\begin{tabular}{|c|c|c|c|c|}
\hline Organ system & ATC code & International proprietary name & Frequency of medicine used & Rank \\
\hline \multirow[t]{3}{*}{ Alimentary tract and metabolism } & $\mathrm{A} 11 \mathrm{~A}$ & Multivitamins & 47 & 2 \\
\hline & $\mathrm{A} 02 \mathrm{~B} \times 02$ & Sucralfate & 41 & 3 \\
\hline & A02BA02 & Pantoprasole & 18 & 7 \\
\hline Blood and blood-forming organs & $\mathrm{B} 01 \mathrm{AB} 06$ & Enoxaparin sodium & 49 & 1 \\
\hline \multirow[t]{2}{*}{ Cardiovascular system } & C03CA01 & Furosemide & 10 & 12 \\
\hline & C09AA04 & Perindopril & 6 & 16 \\
\hline Dermatologicals & H02AB09 & Hydrocortisone systemic & 8 & 14 \\
\hline \multirow[t]{6}{*}{ Anti-infectives for systemic use } & J01CR05 & Piperacillin/tazobactam & 22 & 8 \\
\hline & J01DA04 & Cefazolin & 13 & 10 \\
\hline & J01CR02 & Co-amoxyclav & 11 & 11 \\
\hline & J01DH02 & Meropenem & 11 & 11 \\
\hline & J01DH51 & Imipenem & 11 & 11 \\
\hline & J02AC01 & Fluconazole & 7 & 15 \\
\hline \multirow[t]{3}{*}{ Nervous system } & N02AG01 & Morphine & 38 & 4 \\
\hline & N05CD08 & Midazolam & 32 & 5 \\
\hline & N02BE01 & Paracetamol oral & 28 & 6 \\
\hline Respiratory system & R03BB01 & Ipratropium bromide/fenoterol & 11 & 11 \\
\hline Various & V06B & Dipeptivan & 5 & 17 \\
\hline
\end{tabular}




\section{Diagnoses}

A total of 71 diagnoses were recorded, with a mean of 1.4 (standard deviation 0.6) diagnoses per patient. It was noted that more than one diagnosis may apply to a single patient.

Trauma was the diagnosis most commonly encountered ( $n=21)$, including motor vehicle accidents and gunshot wounds. The injuries ranged from fractures to soft-tissue lacerations and abdominal wounds obtained from gunshot wounds or blunt-force trauma. Most trauma patients admitted also had skeletal or gastrointestinal involvement. Another common diagnosis was gastrointestinal sepsis $(n=11)$ after general surgery.

The diagnosis of infection was made secondary to the admission diagnosis in 14 of the 15 (93\%) cases. This included ventilator-associated pneumonia as well as surgical wound infections.

\section{Prescribed medication}

The medication used was classified according to the Anatomical Therapeutic Chemical (ATC) classification system. A total of 529 medicines were prescribed (Table 1). An average of 12 medicines was prescribed per patient. Medicines most frequently used were for the alimentary tract (174 items (according to the ATC system); 30\%), the anti-infective system (124 items; $21 \%$ ) and cardiovascular system (31 items; 54\%). Medicine from the blood and blood-forming organ system, namely enoxaparin, was prescribed for $49(8.5 \%)$ patients.

\section{Interventions}

A total of 181 interventions was suggested for the 51 study patients (median 4; range 1 - 28 interventions per patient). A total of 127 interventions was accepted and implemented by medical and nursing staff in the unit (Table 2). 'Untreated medical conditions' were addressed most frequently (28 interventions, of which 9 were accepted; $15.5 \%)$. Reminding doctors to prescribe patients' chronic medications, e.g. antihypertensives, and suggesting antidepressants for long-term patients were included in this category.

This was followed by 'length of course or therapy appropriate' (25 interventions, of which 20 were accepted; $13.8 \%$ ). This category included discontinuing antibiotics after completing the course, stopping antibiotic prophylaxis after $24 \mathrm{~h}$ and stopping pantoprasole continuous infusion for gastric bleeding after $72 \mathrm{~h}$, as per hospital protocol.

Table 2. Most frequent types of interventions

\begin{tabular}{|c|c|c|c|}
\hline Most frequent type of interventions & $\begin{array}{l}\text { Interventions } \\
(N=181), n\end{array}$ & Percentage & $\begin{array}{l}\text { Percentage } \\
\text { accepted }\end{array}$ \\
\hline Untreated medical conditions & 28 & 15.5 & 61 \\
\hline Investigations indicated/outstanding & 22 & 12.2 & 32 \\
\hline $\begin{array}{l}\text { Prescribed doses and dosing frequency } \\
\text { appropriate }\end{array}$ & 20 & 11.0 & 80 \\
\hline Length or course of therapy & 25 & 13.8 & 80 \\
\hline Due to system error or non-compliance & 18 & 9.9 & 83 \\
\hline $\begin{array}{l}\text { Factors hindering achievement of } \\
\text { therapeutic effect }\end{array}$ & 10 & 5.5 & 70 \\
\hline Doses scheduled to maximum effect & 9 & 4.9 & 66 \\
\hline $\begin{array}{l}\text { Comparative efficacy of chosen } \\
\text { medication }\end{array}$ & 8 & 4.4 & 50 \\
\hline Therapy tailored to individual patient & 5 & 2.8 & 100 \\
\hline $\begin{array}{l}\text { Route/dosage form or mode of } \\
\text { administration }\end{array}$ & 6 & 3.3 & 100 \\
\hline
\end{tabular}

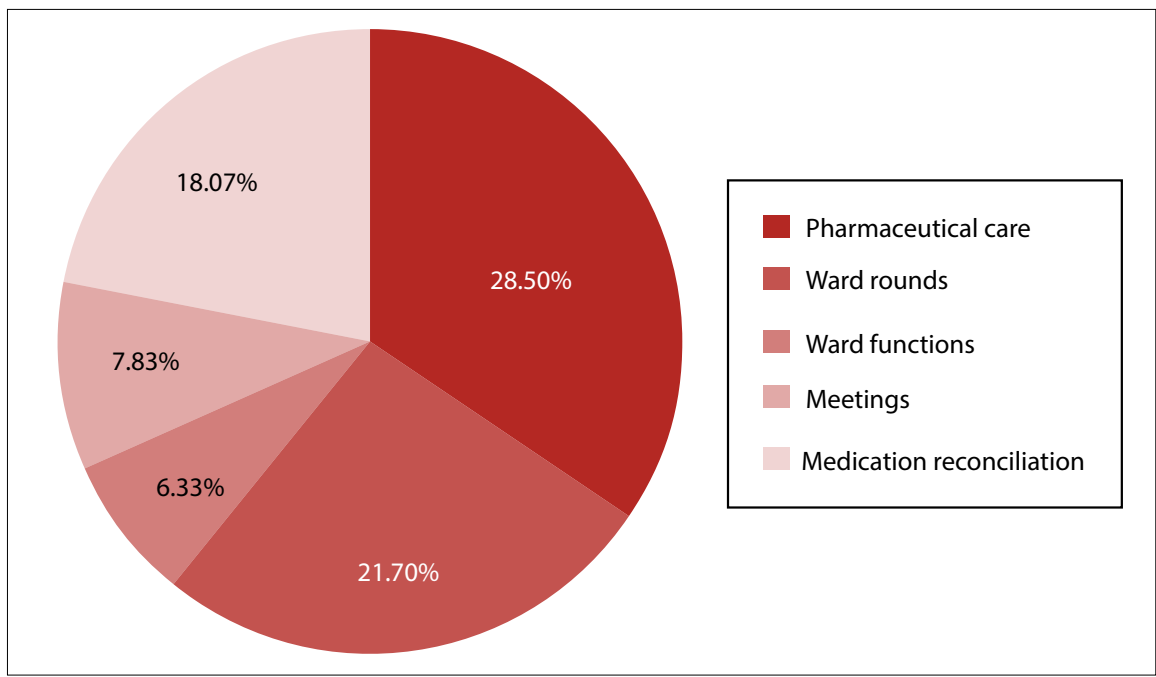

Fig. 1. Percentage of time spent in the ward, total $250 \mathrm{~h}$.

'Investigations outstanding' made up $22(12.2 \%)$ interventions. Only seven of the interventions were successful, because some culture results were never received from the laboratory. Some patients were discharged from the unit before results for serum concentrations of phenytoin were received.

Interventions made to address system errors or non-compliance and factors hindering therapeutic effect totalled 10 (5.5\%) and $5(2.125 \%)$, respectively. These included medicine not received from the pharmacy because of out-of-stock situations, and incorrect doses or medicine not administered to the patient by the nursing staff. Interventions were made during ward rounds with the treating doctors. The pharmacist did a pharmaceutical care round before the ward round, to familiarise herself with the clinical status of each patient. Interventions on drug therapy problems could then be discussed with the treating doctor during ward rounds. The interventions were either accepted or rejected immediately. Interventions made by the pharmacist concerning problems with the pharmacy or counselling of patients or nursing staff occurred after the ward round.

\section{Time spent providing pharmaceutical care services}

The total time spent providing pharmaceutical care services to the ICU was $250 \mathrm{~h}$. Fig. 1 depicts the specific time spent on differentactivitiesin theward.Pharmaceutical care rounds included reviewing the patient's prescription chart, vital signs and laboratory 
results. This took up the majority of time in the ward $(71 \mathrm{~h} ; 28.5 \%)$. Using information obtained during the pharmaceutical care rounds, interventions were suggested on the ward rounds. In some instances, more than one ward round was done per day because of different specialties doing rounds. Ward rounds took up $21.7 \%$ (54 h) of the pharmacist's time.

Medication reconciliation ( $45 \mathrm{~h}$ ) was done by evaluating the patient's prescription chart, and monitoring the medication orders from the pharmacy and the dosing of the different medicines. The pharmacist checked that the correct medicine was received from the pharmacy, and that administration to the patient was correct.

\section{Cost implications}

A direct cost saving due to interventions made by the clinical pharmacist was calculated as R14 353. Having a pharmacist as a permanent member of the multidisciplinary team may be a costsaving opportunity for the unit.

\section{Discussion}

The male/female ratio in the ICU was 2.18:1, which was not a reflection of the general population. According to Statistics $S A,{ }^{[11]}$ the male/ female ratio in 2011 was 0.94:1. The mean age of patients admitted to the surgical ICU was 44.92 years, which is lower than that in the USA or the UK. ${ }^{[12]}$

Trauma, including gunshot and motor vehicle accident injuries, comprised the most frequent diagnoses. This was expected, since it is a surgical and trauma ICU. Nosocomial pneumonia and surgical wound infections were also prevalent as secondary diagnoses. Nosocomial pneumonia has been confirmed in the literature as common in patients undergoing mechanical ventilation. ${ }^{[13]}$

As there was a variety of medications used in the ICU, prescribed from short to more extensive periods, there were many opportunities for the clinical pharmacist to provide pharmaceutical care. The functions that the researcher performed correlated with the scope of a clinical pharmacist in a similar study by Bates et al. ${ }^{[14]}$ Interventions were made regularly to address a variety of medicine-related problems, inappropriate dose or dosing frequency, relative safety of chosen medicine and other factors hindering achievement of therapeutic effect, such as non-compliance and system errors. Similar studies performed in SA also highlighted the abovementioned interventions as being made most frequently by the clinical pharmacist. ${ }^{[9,10,15]}$

The cost of antibiotics and other medicines used by patients the day before and the day after an intervention was calculated. Interventions made included discontinuation of antibiotics after completing a course, or reducing the dose of antibiotics according to guidelines. This amount was a direct calculation and only for the cost of one day after the intervention was performed. No calculations were made for indirect cost implications or length of ICU stay. The cost saving during the study period added up to R14 353 , but potentially could be more. Begley ${ }^{[15]}$ described the practice of pharmaceutical care as having benefits for the healthcare system, including cost saving. Having a pharmacist as a permanent member of the multidisciplinary team may be both a costsaving and extra quality assurance opportunity for the unit. ${ }^{[1]}$

\section{Study limitations}

The study period was very short and considerable time was used to become familiar with the surroundings and routines in the unit. More time was also needed to establish good relationships with attending physicians and medical staff. Pharmaceutical services were rendered only from Monday to Friday, so patients admitted over weekends were not enrolled.

\section{Conclusion}

The study focused on the provision of pharmaceutical care in an ICU and the role of the pharmacist in assessing prescribing patterns, recognising and recording drug-related interventions and the time needed to provide pharmaceutical care.

Interventions were required across a broad spectrum of drugrelated problems. Interventions suggested during ward rounds supplied a good platform for discussions regarding the use of antimicrobials and other medicines, or specific doses of medicine.

The number of medicines prescribed to the patients admitted to the unit during the study period provided an opportunity for the pharmacist to perform numerous interventions, and the conclusion can be made that the continued presence of a pharmacist in the ward would be beneficial to patient outcomes. The provision of pharmaceutical care has the potential to improve the quality of pharmacotherapy and save medicine costs.

After a 2-month period, it was established that there were enough tasks to warrant a full-time position for a clinical pharmacist in the ICU setting.

\section{Recommendations}

A permanent pharmacist should be appointed to the unit to be able to provide a consistent level of care to patients in the ward. A resident clinical pharmacist may also assist in the development and implementation of guidelines for the ward.

Acknowledgements. The authors would like to acknowledge Prof. Herman Schoeman for the statistical analysis of the data and the Department of Pharmacy, Medunsa campus, for financial and logistical support.

References

1. Hepler CD, Strand LM. Opportunities and responsibilities in pharmaceutical care. American Journal of Hospital Pharmacy 1990;47:533-543.

2. American Society of Health-System Pharmacists. ASHP long-range vision for the pharmacy work force in hospitals and health systems. American Journal of Health-System Pharmacy 2007;64(12):1320-1330.

3. Strand L. Building a practice in pharmaceutical care. Pharm J 1998;260:874-876.

4. Munroe WP, Dalmady-Israel C. The community pharmacist's role in disease management and managed care. International Pharmaceutical Journal 1998;12(Suppl 2).

5. Van Mil F. Proving the benefits of pharmaceutical care. Pharmacy World and Science 2004;26:123.

6. Schellack N, Gous AGS. An overview of the time needed to render critical ward services in a neonatal intensive care unit: Documenting the activities of a clinical pharmacist. South African Pharmaceutical Journal 2011;78(7):1-3.

7. Weller TMA, Jamieson CE. The expanding role of the antibiotic pharmacist. Journal of Antimicrobial Chemotherapy (online) 2004;54(2):295-298. http://jac.oxfordjournals.org/cgi/ reprint/54/2/295. 9 (accessed August 2010)

8. American Society of Hospital Pharmacists (ASHP). (1992). Clinical Skills Program: Advancing Pharmaceutical Care. Bethesda. http://www.ashp.org.com (accessed June 2010).

9. Schellack N, Gous AGS. An assessment of the need for pharmaceutical care in an NICU in South Africa. PhD (Pharmacy) thesis. Saarbruken: Lambert Academic Publishing, 2010.

10. Untiedt SM. The impact of pharmaceutical care provided by Medunsa/Technikon Pretoria BPharm IV students at Ga-Rankuwa Hospital. MSc (Med) (Pharmacy) thesis. Dissertation. Pretoria: University of Limpopo, 2004.

11. Statistics South Africa. Midyear population estimates, 2011. www.statssa.gov.za/publications/ P0302/P03022011.pdf (accessed September 2011).

12. Wunsch H, Angus DC, Harrison DA, Linde-Zwirble WT, Rowan KM. Comparison of medica admissions to intensive care units in the United States and United Kingdom. Am J Respir Crit Care Med 2011;183(12):1666-1673. [http://dx.doi.org/10.1164/rccm.201012-19610C]

13. Cunha BA. Pneumonia Essentials. 2nd ed. Royal Oak, Michigan, USA: Physicians Press, 2008.

14. Bates DW, Spell N, Cullen DJ. Systems analysis of adverse drug events. JAMA 1995;274(1):35-43.

15. Begley A. Pharmaceutical care in private hospital intensive care unit. Magister Pharmaciae dissertation. Port Elizabeth: Nelson Mandela Metropolitan University, 2006. 